\title{
Redéfinir le problème climatique par l'écoute du local : éléments de propédeutique
}

\author{
Hervé Brédif ${ }^{1}$, François Bertrand ${ }^{2}$, Martine Tabeaud ${ }^{3}$ \\ 1 Géographie et biogéopolitique, Université Paris 1 Panthéon-Sorbonne, UFR de Géographie, UMR 7533 LADYSS, 75005 Paris, \\ France \\ 2 Aménagement de l'espace et urbanisme, Université de Tours, CNRS, UMR 7324 CITERES, 37204 Tours cedex 3, France \\ 3 Géoclimatologie, Université Paris 1 Panthéon-Sorbonne, UFR de Géographie, UMR 8185 ENeC, 75013 Paris, France
}

Mots-clés :

problème climatique ; découplage science/ politique ; articulation global-local ; problème à double visage ; propédeutique

\begin{abstract}
Résumé - Assimilés à de l'inaction, les résultats décevants du processus international de négociation sur les changements climatiques sont régulièrement interprétés comme la conséquence d'un découplage caractérisé entre science et politique. Les observations concrètes et les analyses que nous avons effectuées en France, dans des situations diverses et auprès d'opérateurs extrêmement variés, invalident cette thèse. Envisageant le changement climatique depuis les territoires et les stratégies locales d'atténuation et d'adaptation, l'article suggère que le problème procède plutôt d'une articulation encore insuffisante entre niveau global et niveau local-territorial d'expertise et de cadrage de l'action. Aussi convient-il de considérer à nouveaux frais le problème climatique, d'en reconnaître et d'en approfondir la double nature ou le double visage, en l'appréhendant dans ses données matérielles et objectives comme dans ses composantes culturelles, symboliques et subjectives.
\end{abstract}

\section{Keywords:}

climate change issues; disconnection between science and politics; local-global fit; dual problem; propaedeutics

\begin{abstract}
Reframing climate issue by listening to local actors: elements of propaedeutic. The disappointing outcomes of international negotiations on climate change are frequently associated with inaction and the consequences of a perceived disconnection between science and politics. However, our experiences and empirical findings based on research carried out in France in highly diverse situations and among very different operators invalidate this thesis. By analysing climate change from the standpoint of territories and local adaptation and mitigation strategies, our article contends that the problem stems instead from a still insufficient fit between local and global levels of expertise and action frameworks. Moreover, we also need to analyse climate change from a new angle and recognise its dual nature in material and objective terms as well as in its cultural, symbolic and subjective components.
\end{abstract}

Depuis le premier sommet de la Terre, la négociation climatique procède fondamentalement d'un principe de responsabilité pensé sur le mode de l'imputation. Imputation nécessitant de pouvoir établir une causalité aussi directe, stable et certaine que possible entre une entité émettrice de gaz à effet de serre (GES) et des effets induits. Conçu selon cette logique, le protocole de Kyoto n'a cessé d'en accuser les limites et les apories : batailles de chiffres (autour des années de référence, des ratios d'équivalence, des périmètres de comptabilisation, etc.) ; clés de répartitions du " partage du fardeau » entre pays et secteurs d'activités suscitant tensions et rejets, avant d'être invalidées par des évolutions économiques et technologiques rapides; comptabilités-carbone devenues fort discutables dans un monde toujours plus interdépendant... C'est pourquoi, sans doute, dans le processus international qui précède la conférence de Paris de fin 2015, des voix s'élèvent pour préconiser un changement radical d'approche: plutôt que de rechercher des responsabilités négatives, actuelles ou passées, et de poursuivre pour seul objectif un engagement ferme des États à réduire leurs émissions de GES, il conviendrait de raisonner davantage en termes d'opportunités offertes par les stratégies bas carbone. Une manière de concevoir la responsabilité de façon positive, en stimulant la contribution volontaire et motivée de chacun pour

Auteur correspondant : H. Brédif, hbredif@univ-paris1.fr 
l'avènement d'un avenir commun plus soutenable (Brédif et Tabeaud, 2013) ?

Il est un domaine cependant où la recherche des coupables occupe toujours les esprits. En 2009, d'une même voix, ONG, experts, médias, intellectuels et observateurs, en nombre, n'ont pas hésité à attribuer aux États la responsabilité de "l'échec» de la conférence de Copenhague. Les politiques, au plus haut niveau, auraient révélé leur incapacité à prendre les décisions qui s'imposaient, quand scientifiques et experts, dans leur grande majorité, estimaient qu'il n'était plus permis de louvoyer. Ce message ou ce schème, depuis, n'a cessé de se faire entendre, au point de devenir la manière habituelle de résumer le problème climatique ${ }^{1}:$ « La science a parlé, mais la politique tarde à agir. » Ainsi se trouve fondée l'idée d'un découplage entre science et politique, qui serait à l'origine de « l'inaction » déplorée par tant de commentateurs.

Plusieurs explications sont classiquement avancées pour rendre compte de cette situation. Selon certains, la faiblesse de l'engagement politique proviendrait du travail de sape effectué par des lobbies économiques qui craignent une remise en cause de leurs positions (Conway et Oreskes, 2014) ; plus encore, elle tiendrait à l'intervention de forces puissantes ayant intérêt à perpétuer le fonctionnement de l'ancien monde plutôt que d'accepter les obligations nouvelles de l'Anthropocène (Latour, 2013). Considérant plus généralement que l'économie constitue le nerf de la guerre, d'autres pointent l'antagonisme potentiellement paralysant entre des priorités aux horizons temporels divergents, entre relance de la croissance et maintien du système climatique dans des limites gérables : ce pourquoi, après bien des rapports similaires, le rapport Stern et Calderon (2014) tente de montrer que la recherche d'une meilleure croissance et d'un meilleur climat peuvent en réalité marcher de concert. Cependant, pour d'autres encore, la médiocre qualité de la transmission entre science et politique relèverait de raisons plus fondamentales: elle se ferait d'autant plus mal que les opinions publiques, sinon le genre humain, auraient finalement des priorités différentes et seraient peu enclines à imaginer le pire (Dupuy, 2004). Une propension à l'évitement aggravée, voire encouragée, par les fondements mêmes de la démocratie représentative, dans son impuissance constitutive à prendre en compte la durée longue (Rosanvallon, 2009), les phénomènes et processus environnementaux globaux, nécessairement transterritoriaux (Bourg et

\footnotetext{
1 Le terme de «problème climatique » désigne dans ce texte le changement climatique au sens de la Convention-cadre des Nations unies sur les changements climatiques (CCNUCC) en tant que problème public, reconnu par les différentes institutions internationales et nationales, et constituant un nouvel objet d'action collective par rapport auquel sont déclinées, à différentes échelles, des politiques publiques.
}

Whiteside, 2010), à reconnaître surtout, outre les intérêts des humains, les exigences des vivants non humains et de cette somme de processus physiques et biologiques que constitue la Terre-globale ou Biogée, notre hôte planétaire (Serres, 2009).

Sans minimiser ces différentes explications, les lignes qui suivent explorent une voie distincte. Une voie nourrie des travaux de terrain et des retours d'expériences que nous accumulons depuis plus de dix ans au contact d'opérateurs variés, publics et privés, œuvrant principalement en France à des échelles et dans des contextes territoriaux contrastés, et ayant en commun de tenter de donner une traduction locale aux politiques et exigences climatiques (voir encadré 1 ).

Les enseignements tirés de ce corpus de cas concrets, qui couvrent des situations extrêmement diversifiées à l'échelle de la France, suggèrent que la formulation du problème en termes de découplage entre science et politique masque en réalité un découplage d'une autre nature, nettement plus incapacitant. Ce dernier se révèle à condition d'envisager le changement climatique depuis les territoires et les stratégies locales d'adaptation et d'atténuation. Il correspond en dernière analyse à une articulation encore insuffisante entre niveau global et niveau local-territorial d'expertise et de cadrage de l'action. Pour le montrer, nous étudierons successivement : la réception du cadrage global de la question climatique au niveau local (point 1), puis les limites rencontrées localement dans la mise en œuvre des politiques climatiques (point 2) ; réciproquement, la difficile reconnaissance des multiples initiatives locales et de leur logique d'existence et de diffusion (point 3). Cela permet de renouveler en profondeur la perspective sur la question du couplage science/société et d'esquisser une reformulation du problème climatique dans son ensemble (point 4). Invitation à se tenir autant à l'écoute des faits et signes stratégiques que des faits scientifiques, la démarche suivie dans cet article présente finalement les différents attributs d'une réflexion propédeutique entendue dans le sens plein du terme.

\section{Pas de déni, mais des obstacles réels à l'appropriation du problème}

\section{Un déni de réalité face à l'énormité du problème climatique?}

Pour les très nombreux spécialistes impliqués dans les débats autour du problème climatique, membres du GIEC, ONG, intellectuels et responsables politiques, la situation actuelle est jugée alarmante. Ils en viennent à penser que l'obstacle se situe au niveau des opinions publiques, qui refusent de voir la menace de la catastrophe et dont l'attitude relève d'un « déni de la réalité ». 
Encadré 1. Des retours d'expériences privilégiant les organisations de petite taille

Prises dans une course au leadership, les villes mondiales et les grandes agglomérations, de l'Europe du Nord en particulier, ne pouvaient négliger la dimension climatique. Dans ce domaine comme dans d'autres, elles rivalisent de volontarisme, d'ingéniosité et bien sûr en puissance de communication, affichant les politiques climatiques les plus ambitieuses, promettant de réduire de manière significative leurs besoins énergétiques, et partant, leur empreinte carbone. Aussi, les études et comparaisons internationales n'ont-elles pas tardé à prendre pour cas d'école ces « bons élèves », élevés au rang de pionniers quand ils n'étaient souvent que les premiers de la classe. Analyses et publications, fort bien documentées, ont suivi (Betsill et Bulkeley, 2004; Corfee-Morlot et al., 2009; Hodson et Marvin, 2010 ; Emelianoff, 2014).

Cependant, comme le rappelle un rapport publié fin 2013 sous l'égide du Comité des régions de l'Union européenne, $46 \%$ des villes ne font que débuter leur plan climat local. Pour nombre de collectivités locales et territoriales, d'organisations publiques et privées plus généralement, la mise en place de stratégies d'atténuation et d'adaptation progresse donc lentement. Ces organisations ne disposent souvent pas des moyens techniques et humains qui leur permettraient de faire une priorité de la définition et du suivi d'une politique climatique dans la durée.

Pour les besoins de cet article, les auteurs ont choisi de privilégier les collectivités de petite taille, qu'elles soient urbaines ou rurales. Aussi s'agira-t-il moins de rappeler ce que plusieurs études internationales confirment déjà que de mettre l'accent sur des difficultés et des limites qui se remarquent mieux auprès des acteurs et des organisations ordinaires. Enfin, le corpus de cas mobilisés se limite au contexte français. Cela peut constituer un avantage, si l'on veut bien considérer que la France est un pays centralisé, où il est de ce fait relativement aisé de suivre la chaîne descendante des politiques et stratégies climatiques définies à de hauts niveaux de décision ; qu'en outre, elle occupe une situation institutionnelle intermédiaire entre les pays du Nord, et les pays de l'Est et du Sud de l'Europe, ces derniers étant souvent moins avancés en matière de politiques climatiques.

Quelque dix années de recherches et de pratiques au contact du terrain président aux considérations développées dans cet article. À eux trois, les auteurs couvrent un champ extrêmement diversifié de situations territoriales, qui vont du rural et du forestier à l'urbain dense, des petites communes aux Régions en passant par les communautés de communes et d'agglomération, les Pays et les parcs naturels régionaux, des petits porteurs de projets individuels à de grandes entreprises. Le référentiel de cas concrets qui en résulte mobilise trois formes d'expertises distinctes et complémentaires : 1 . Des études et des enquêtes relatives à des politiques territorialisées d'atténuation et d'adaptation réalisées dans plusieurs régions (Nord-Pas-de-Calais, Poitou-Charentes, Réunion, Rhône-Alpes) et communautés de communes (Bertrand et Larue, 2007 ; Bertrand et Rocher, 2007 ; Bertrand et al., 2012) ; 2. Un appui direct à des opérateurs publics et privés dans l'élaboration de plans climat territoriaux (pour notamment une quinzaine de collectivités territoriales des régions Île-de-France, Picardie et Centre, ainsi que trois grandes entreprises) ; 3. Plusieurs centaines d'entretiens approfondis réalisés dans le cadre de démarches de facilitation (plateau de Saclay, Hautes-Pyrénées) ou de recherches-actions (plateau de Millevaches, forêt de Tronçais, Médoc, Haut-Allier) permettant d'apprécier la place accordée par les acteurs et responsables locaux aux questions de changement climatique dans leurs stratégies et projets de développement.

Ce «syndrome de Cassandre » leur paraît constituer la cause ultime et première de l'inaction ; les freins seraient donc d'ordre intellectuel et cognitif. Ainsi, l'éthicien Clive Hamilton (2013, p. 138) résume-t-il un diagnostic largement partagé lorsqu'il déclare : « Le déni des sceptiques a certes réussi à brouiller la compréhension de l'opinion, mais les stratégies communément utilisées par le public pour éviter ou pour sous-estimer les avertissements des scientifiques ont été un facteur plus puissant encore de la réticence des gouvernements à agir en conséquence. » Avant de conclure : « Nous sommes tous des climato-sceptiques » (2012). En somme, cette thèse du déni étendu à tous les aspects du changement climatique, régulièrement formulée dans le langage de la psychologie comme "dissonance cognitive caractérisée ", considère que le problème climatique est victime $\mathrm{d}^{\prime} \mathrm{un}$ rejet dès son énonciation : nous n'agissons pas, car tous nous refusons de croire au message - nous n'y adhérons pas fondamentalement - ou bien nous refusons de le faire nôtre - nous ne nous l'approprions pas vraiment. Il convient de voir si cette thèse, qui joue pour beaucoup dans l'idée d'un découplage entre science et politique, se confirme sur le terrain.

\section{Une large reconnaissance du problème climatique}

En pratique, cependant, l'existence du changement climatique est globalement et de plus en plus largement admise. Les multiples marches, actions et camps « pour le climat» témoignent d'une reconnaissance sociale croissante du problème - à l'image de la dernière en date, la People Climate's March du 21 septembre 2014 
pour laquelle des centaines de milliers de citoyens se sont mobilisés dans le monde à l'occasion du sommet sur le climat de New York. En France, comme le montrent les enquêtes de l'Ademe (2013), 60 \% des personnes souscrivent à l'affirmation selon laquelle «l'augmentation de l'effet de serre [qui] entraînerait un réchauffement de l'atmosphère de la terre [...] est une certitude pour la plupart des scientifiques » et $70 \%$ souscrivent à l'affirmation selon laquelle « le réchauffement de la planète est causé par les activités humaines ».

Cette reconnaissance n'est évidemment pas étrangère au fait que l'immense majorité des experts et des scientifiques admet sans difficulté la réalité du réchauffement climatique. Pour autant, cela n'exclut pas au sein de la communauté des chercheurs des points divers de controverse, souvent mal compris par le grand public. Citons, entre autres, des remarques méthodologiques sur les calculs des moyennes, sur les reconstitutions de séries antérieures grâce à divers marqueurs, sur la fiabilité de modèles planétaires qui ne peuvent inclure toutes les interactions et rétroactions d'un système complexe, etc. D'autres critiques s'attachent aux causes du réchauffement et à l'estimation de la part anthropique par rapport aux causes naturelles, possiblement sous-estimées. $\mathrm{Au}$ total, s'il existe bien des "marchands de doute" (Oreskes et Conway, 2010) et des « semeurs de trouble » (Godard, 2012), les contradicteurs, pour la plupart, s'attaquent à ce sujet multidisciplinaire et complexe par des entrées différentes compte tenu de leur culture scientifique et sans remettre en question l'existence même du réchauffement global. Les aspects qui alimentent le scepticisme, au demeurant essentiel au progrès scientifique, ne permettent donc pas de justifier une certaine rhétorique du « déni en bloc ».

L'observation des différentes initiatives locales conduites en France atteste que le changement climatique est largement reconnu comme un problème nécessitant des réponses en termes d'actions de la part des acteurs publics. Rarissimes, voire inexistantes, sont les remises en cause quant à l'existence du changement climatique; quant à son origine anthropique, elle n'est guère discutée. Les nombreux relais d'opinions et les multiples campagnes sur le changement climatique ( « défi climat », « familles à énergie positive », etc.) montrent que l'existence $\mathrm{du}$ problème climatique n'est pas contestée par les acteurs locaux.

\section{Les caractéristiques du problème retardent son appropriation}

L'appropriation locale n'en reste pas moins délicate. Le problème climatique demeure une construction, un grand récit scientifique, assez largement déconnecté de la vie quotidienne des habitants (au contraire d'autres problèmes locaux comme les déchets, les pollutions de l'eau ou de l'air, etc.). Immatérialité, imprévisibilité des seuils de basculement, irréversibilité du phénomène: une difficulté majeure dans l'appréhension des changements climatiques en tant que réalité palpable et problématique réside sans doute en grande partie dans l'impossibilité d'établir un lien entre les pratiques et les actes commis à l'échelle locale et leurs conséquences sur le climat à la même échelle.

Pour les collectivités territoriales, il s'agit en quelque sorte de résoudre un problème planétaire avec des réponses nécessairement limitées aux compétences et aux limites spatiales de chaque collectivité. La divergence apparaît alors maximale entre le périmètre de construction du problème, planétaire, et les périmètres de résolution, limités aux territoires d'action des collectivités locales. Ce décalage spatial se double d'un décalage temporel lié à l'inertie des phénomènes en cause. L'incommensurabilité entre l'échelle spatiotemporelle $\mathrm{du}$ problème et les moyens de changements a priori disponibles constitue un frein majeur à l'action individuelle et collective.

Or, avant de débattre localement des implications et des réponses envisageables, acceptables, désirables face au problème climat, il faudrait d'abord que ce problème existe socialement. Et actuellement, il n'existe guère de personnes en mesure d'être des porte-paroles locaux du climat (à l'inverse des questions sur la qualité de l'eau ou sur la biodiversité par exemple). «Au niveau local, les controverses sociotechniques sur le climat n'en sont qu'à leurs prémices. Comparativement à d'autres situations de gestion de l'environnement [...], les positions des différents acteurs ne sont pas encore constituées» (Petit, 2011, p. 103). La construction sociale du problème climat reste encore largement à faire aux échelles locales. Elle suppose une communauté d'intérêts qui n'émerge pas spontanément, les habitants ayant d'autres problèmes plus manifestes et plus faciles à identifier autour desquels se rassembler. En cela, ce problème s'inscrit bien dans la modernité, telle que l'analyse Giddens (1994, p. 27) : les individus se retrouvent à entretenir des relations avec un autrui « absent » de toute situation de face-à-face. «Le rapport à l'environnement se trouve inscrit de fait dans une "communauté globale"[...]» (Petit, 2011, p. 116).

\section{Les limites d'une approche top-down reposant sur des stratégies dédiées}

\section{La lente mais réelle percolation française}

La Convention-cadre des Nations unies sur les changements climatiques (CCNUCC) a mis en place dès 1992 un cadre intergouvernemental pour faire face au défi 
posé par les changements climatiques. En 2000, la France adopte un programme national de lutte contre le changement climatique (PNLCC), qui engage le pays à ramener en 2010 ses émissions de GES à leur niveau de 1990, conformément au protocole de Kyoto de 1997. Ce plan est conçu exclusivement dans une optique de réduction des émissions de GES. Le rapport liste donc successivement les divers secteurs d'activité fortement émetteurs de GES : les transports, les bâtiments, l'industrie, l'énergie et les déchets, l'agriculture et les forêts. Avec quelques modifications, les plans de 2006, 2009 et 2011 reprennent ce découpage sectoriel bien adapté à la ventilation des quotas. Seul l'intitulé change en 2014 : « Politiques climat et efficacité énergétique », afin de se démarquer du Plan national d'adaptation au changement climatique (PNACC), adopté en juillet 2011. Ce plan comporte 200 recommandations classées en fichesactions thématiques: santé, ressource en eau, biodiversité, risques naturels, agriculture, forêt, pêche, énergie et industrie, transport, bâti, tourisme, information, éducation, recherche, etc. L'approche demeure sectorielle, à l'exception de deux chapitres «littoral » et «montagne» et d'une approche «ville» en filigrane dans le chapitre " urbanisme et cadre bâti ».

Cependant, dès 2002, les engagements semblent déjà impossibles à atteindre. Malgré une baisse légère des émissions dans l'industrie et une stabilisation dans le chauffage des bâtiments suite à un hiver doux, on observe une augmentation pour la production d'électricité et dans les transports. Aussi, par souci d'efficacité, le Plan climat national de 2004 invite-t-il les collectivités à décliner l'action nationale à une échelle plus fine, via des "plans climat territoriaux", rebaptisés "plans climat énergie territoriaux » (PCET), suite à la loi « Grenelle 2 » (juillet 2010). Cette même loi rend l'élaboration de ce plan obligatoire pour les régions, les départements, les communautés urbaines, les communautés d'agglomération, ainsi que pour les communes et communautés de communes de plus de 50000 habitants. En deçà de ce seuil de population, les territoires peuvent en adopter un à titre volontaire. Le PCET doit constituer un projet spatialisé de développement durable, déclinant concrètement l'objectif des $《 3 \times 20 \% »^{2}$ du plan climat de l'Union européenne, grâce à un responsable qui identifie les risques et les opportunités locales et qui trie les priorités d'action dans une stratégie à moyen terme. Plusieurs guides ont été mis à la disposition des collectivités

\footnotetext{
2 Adopté en décembre 2008, cet objectif des 3 × $20 \%$ consiste à porter à $20 \%$ les énergies renouvelables dans le mix énergétique européen, à réduire de $20 \%$ les GES des pays de l'Union et à accroître de $20 \%$ l'efficacitéénergétique d'ici à 2020. Au passage, cela montre assez que, dans un PCET, la question énergétique est prépondérante, tandis que la notion de climat se trouve souvent réduite à sa plus simple expression et comme confondue avec une teneur en GES.
}

afin de les accompagner. Le couplage science/politique a donc fonctionné à plein. La dynamique de territorialisation du "problème climat » a suivi, en France, une institutionnalisation descendante ; elle n'est pas le fruit $\mathrm{d}^{\prime}$ une action ascendante des collectivités et ne résulte pas des besoins spécifiques de celles-ci par rapport à d'éventuels effets locaux des changements climatiques (Bertrand, 2013).

\section{Les PCET rapidement confrontés à de sérieuses limites}

La loi impose peu de choses quant au contenu du PCET, si ce n'est un «diagnostic carbone » assez sommaire ${ }^{3}$. Malgré son caractère quantitatif, le décompte des émissions de GES est marqué par l'imprécision ${ }^{4}$ (MIES, 2006) et une comparabilité réduite entre territoires ${ }^{5}$. Au demeurant, les diagnostics carbone technicisent les débats, mais ne s'avèrent guère parlants pour le citoyen ordinaire. Quant aux objectifs quantifiés de réduction des émissions de GES autour desquels s'organisent les PCET, ils sont très souvent définis dans une logique mimétique et a-territoriale (en reportant de façon homothétique un objectif décidé à un niveau supérieur et sans le modérer suivant les caractéristiques locales) ${ }^{6}$, hors de tout débat, et parfois dans des logiques de pur affichage ou de surenchère politique. La mesure des résultats à l'aune de ces objectifs de réduction souligne bien souvent la disproportion entre l'ampleur du problème et les moyens mis à disposition. De sorte que la focalisation sur ces objectifs quantifiés finit par s'avérer largement contre-productive. En effet, ces objectifs apparaissent tellement hors de portée, qu'ils découragent les élus de poursuivre les actions engagées et de reconduire les budgets nécessaires à cet effet et le grand public de

\footnotetext{
3 Cette obligation légale est restreinte aux «patrimoine et compétences » de la collectivité (art. 75, loi Grenelle 2).

4 En l'absence de données mesurées, les valeurs sont calculées par agrégation d'estimations et de valeurs moyennes (en matière d'alimentation, d'habitat, de mobilité, etc.) disponibles au niveau régional, voire national, converties selon des facteurs d'émissions. De plus, les chiffres officiels s'appliquent aux seules émissions, pas aux flux de GES. Ainsi, en France, de 1990 à 2007, le niveau moyen individuel d'émissions de GES sur le territoire a diminué de $15 \%$, alors que l'empreinte carbone par individu a augmenté de $5 \%$ sur la même période (CGDD/ SOeS, 2012).

5 Les choix des années et des périmètres de référence s'avèrent cruciaux lorsqu'il s'agit de mesurer l'évolution des émissions des territoires dans le détail.

6 Il est très rare d'observer la définition d'objectifs en fonction des enjeux, sources et gisements territoriaux en matière de GES. La pratique courante consiste à reprendre un objectif national (du « facteur 4 » pour 2050) ou européen (des « $3 \times 20$ » pour 2020), sans l'ajuster aux caractéristiques du territoire.
} 
continuer à faire des efforts. "Il semble exister un gouffre entre l'investissement politique nécessaire pour résoudre la crise climatique et les marges de manœuvre à la disposition des élus » (Godinot, 2011). Ce découplage entre moyens et objectifs n'est pas propre à l'action locale et se retrouve aux autres niveaux d'organisation, comme l'attestent les effets d'annonce des gouvernements avec des objectifs toujours plus ambitieux ${ }^{7}$, mais une conduite désordonnée ${ }^{8}$.

Pour les instances locales de décision, le nouvel horizon planétaire dans lequel doit s'inscrire leur action produit un décadrage. Elles se retrouvent en effet confrontées « à une multiplicité d'enjeux relatifs à l'articulation des échelles spatio-temporelles, qui retentissent sur les logiques d'implantation des activités ou de l'habitat, les modes de mobilité, l'approvisionnement énergétique, les choix des matériaux, etc. » (Emelianoff, 2004). Cela correspond en fait à un renversement de perspective : les politiques climatiques locales s'appuient sur une mise à disposition de moyens locaux au bénéfice de problématiques globales, ce qui tranche avec les politiques d'aménagement traditionnellement pensées comme une mise à disposition de moyens extraterritoriaux au bénéfice de problématiques locales. Ainsi, les périmètres de références de certaines politiques locales (le bassin versant, le massif montagneux, etc., voire l'atmosphère planétaire) ne correspondent plus aux périmètres habituels de la légitimité politique, à savoir les circonscriptions électorales.

\section{Place du référent climat et outils de diagnostic sont des aspects critiques}

La mise en œuvre d'actions locales face au «problème climat " reconduit le même dilemme organisationnel que pour l'application du développement durable : doit-on privilégier des politiques spécifiques, en leur dédiant un service ou une direction, nécessairement isolés et opérant rapidement à contre-courant de l'institution ou doit-on préférer l'installation dans chaque service d'un référent « énergie-climat » ? La mission énergie-climat doit-elle être intégrée à la direction en charge de l'environnement, des moyens généraux, ou

\footnotetext{
7 En octobre 2014, les députés européens ont porté les objectifs contraignants à au moins $40 \%$ de réductions d'émissions de GES, au moins $40 \%$ d'efficience énergétique, avec au moins $30 \%$ de renouvelables d'ici à 2030.

8 Dans son rapport La mise en œuvre par la France du Paquet énergie-climat (2014), la Cour des comptes souligne l'illisibilité de la politique énergie-climat, tant sur le plan européen que dans sa transcription nationale, du fait notamment de la multiplicité des textes de références et des horizons $(2020,2050 \ldots)$ et de l'hétérogénéité des objectifs (développement des énergies renouvelables, économies d'énergie et baisse des GES).
}

bien rattachée plus haut dans l'organigramme? Il est évidemment difficile de trancher, tant les situations sont variées et évolutives, mais il s'agit pourtant d'un point critique. Dans tous les cas, il est essentiel que le référentclimat ne se retrouve pas isolé et puisse échanger avec les collègues des différents services de son organisation, eu égard aux nombreuses problématiques avec lesquelles interagit la question climatique ; en outre, l'élaboration d'un plan climat digne de ce nom demande de la continuité (plusieurs années sont nécessaires) et de la stabilité dans le poste.

Quand les objectifs de réduction de GES peuvent paraître difficilement atteignables, voire démotivants pour les agents chargés de mettre en œuvre localement les politiques climatiques, des démarches tel le diagnostic Cit'ergie ${ }^{9}$ suscitent davantage d'adhésion en raison de leur pertinence. Ce diagnostic procure une visibilité sur un champ de compétence « énergie-climat » relativement nouveau pour les collectivités et permet de privilégier un nombre restreint de mesures, jugées prioritaires, au regard des marges de progrès et des actions déjà entreprises par la collectivité. En ce sens, il entre bien dans la logique des mesures dites "sans regret », habituellement associées aux stratégies d'adaptation.

Malgré les limites et difficultés évoquées ci-dessus, l'intérêt d'une politique dédiée, en termes de visibilité et de symbole, parait relativement clair. Un premier plan climat local permet de relire l'ensemble des actions de la collectivité sous le prisme des économies d'énergie, ce qui amène à rassembler, harmoniser, compléter et coordonner différentes mesures et sous-mesures qu'avaient déjà prises la collectivité ou l'organisation, mais qui demeuraient éclatées. Cette expérience peut être culturellement nécessaire et elle peut permettre de trouver des synergies jusqu'alors peu remarquées.

\section{L'effervescence des initiatives}

\section{Un mouvement de fond considérable}

La volonté de parvenir à une réponse globale face aux menaces du changement climatique, au travers d'un cadre institutionnel unifié privilégiant de grands acteurs (États, GIEC, organes onusiens, etc.), a absorbé beaucoup d'énergie et accaparé les esprits. Elle a détourné l'attention d'un mouvement de fond multiforme, dont on repère au mieux quelques éléments saillants quand une association de villes globales ou bien des marques réputées réussissent à faire valoir le

\footnotetext{
9 Le label Cit'ergie est la déclinaison française du label européen « Energy Award ». Il récompense les collectivités engagées dans un processus de management de la qualité, appliqué à la mise en œuvre de leur politique de l'énergie.
} 
sérieux de leur engagement vis-à-vis du climat. Mouvement qui doit sans doute autant à la pression croissante exercée par les rapports du GIEC qu'aux avancées incertaines de la négociation internationale. Mouvement difficile à saisir, tant il s'incarne dans des entités nombreuses - collectivités locales, entreprises de toutes sortes, associations citoyennes, ainsi que dans d'autres, plus improbables, tels des groupes d'artistes, des congrégations religieuses, de simples individus aussi - et tant il revêt des formes d'engagement diversifiées. Mouvement si considérable cependant qu'il importe d'en esquisser les traits essentiels, sans bien entendu pouvoir prétendre à ce stade à une quelconque exhaustivité.

\section{La réduction des GES ne constitue pas la motivation première}

Une des caractéristiques manifestes de cette multitude d'initiatives locales ${ }^{10}$ réside dans ce qu'elles affichent d'autres préoccupations et poursuivent d'autres objectifs que ceux habituellement liés au changement climatique, même si celui-ci n'est pas oublié (voir encadré 2). En fait, elles semblent obéir à un nombre limité de motivations, l'énergie et la santé en constituant aujourd'hui les deux plus remarquables. La première, dans un souci de réduction de la facture énergétique ou de la dépendance aux énergies fossiles, va souvent de pair avec une recherche accrue d'autonomie ; dans certains cas, elle est manifestement sous-tendue par une vision de la résilience locale qui n'est pas sans rappeler les idées développées par Hopkins dans son fameux Manuel de transition (2010). La seconde se situe aux fondements de politiques locales visant à lutter contre la pollution de l'air ambiant, extérieur ou intérieur: en Chine, ainsi que dans de nombreux pays, l'amélioration de la qualité de l'air de proximité fait figure de grande cause et sert de levier de sensibilisation et d'action vis-àvis de la question climatique en général ; ailleurs, elle peut revêtir les habits du bien-être : à Montréal, l'incitation à recourir à la marche et au vélo - et donc à réduire les émissions de GES - met en avant des gains en matière de santé et de qualité de vie (lutte contre le surpoids, contribution neutre à la pollution atmosphérique, etc.). D'autres grands leviers d'action gagnent visiblement en consistance et en force : la réduction des vulnérabilités aux risques naturels et technologiques et la résilience des sociétés et des environnements; la relocalisation des productions et des emplois, et l'avènement d'un nouveau modèle économique, voire d'une nouvelle forme

\footnotetext{
10 Les politiques climatiques mises en place par les collectivités relèvent plutôt de la déclinaison des stratégies et politiques globales (seconde partie de l'article). Elles n'entrent donc pas à proprement parler dans ce que nous appelons ici des « initiatives locales ».
}

\section{Encadré 2. Que faut-il entendre par local ?}

La notion de local ne doit pas s'entendre exclusivement dans un sens géographique, administratif et territorial renvoyant au "lieu de petite extension » comme l'examen des stratégies top-down aurait pu le laisser penser. Il convient premièrement de noter que « la taille de la localité varie par rapport à celle du global à laquelle on la réfère » (Lussault, 2003). Dans une perspective continentale ou mondiale, le local peut très bien s'apparenter à la région, voire au pays, alors que dans une perspective nationale ou régionale, il s'agira plutôt de l'aire urbaine ou de la commune. En second lieu, il faut également l'envisager au sens de la topologie de l'espace, le local correspondant alors à un point spécifique d'un réseau, susceptible, pour le sujet qui nous intéresse, de correspondre en dernière instance à un individu précis.

de société à la fois plus solidaire et plus conviviale ; la réduction de l'empreinte écologique et la conservation de la nature et de la biodiversité.

$\mathrm{Au}$ regard du changement climatique, ces initiatives relèvent donc d'une philosophie de l'action bien spécifique. Premièrement, la contribution à la résolution du problème climatique fait souvent partie explicite de la donne, mais n'intervient que comme motif ou comme gain supplémentaire, induit ou collatéral ; elle ne constitue pas le moteur principal de l'action. En conséquence, ces initiatives sont difficiles à classer, et plus encore à comptabiliser. Elles présentent en effet une pluralité de cobénéfices, alliant immédiateté et long terme, retombées individuelles (dépenses, santé, etc.) et intérêt général, jouant à la fois sur l'adaptation et la résilience, cumulant la recherche d'une réduction des GES et d'une amélioration de plusieurs paramètres de la qualité de la vie. Enfin, elles poursuivent des finalités clairement positives. Des exemples? L'Association nationale pour la prévention de la qualité de l'air (Respire) se donne pour objectif de lutter contre la concentration en ozone, non dans la stratosphère, mais dans l'air que chacun inhale et qui, au-delà d'un certain seuil - les fameux pics d'ozone -, nuit gravement à la santé des plus faibles, notamment les enfants sensibles à l'asthme. Abaisser le taux de pollution suppose de réduire la consommation de carburants, et contribue ainsi à diminuer les émissions de GES, mais ce dernier aspect est présenté comme un avantage subsidiaire. Les initiatives privées ou associatives de covoiturage et d'autopartage, dopées par les technologies de l'information et de la communication, redessinent le paysage des transports et présentent de nombreux avantages pour leurs utilisateurs, avec de surcroît une réduction appréciable des émissions de GES. Quant aux boisements effectués par des forestiers (pour 
des raisons économiques, en réponse aux besoins de matériaux de construction, etc.) ou par certaines agglomérations (pour les activités récréatives de citadins, le cadre de vie, etc.), leurs promoteurs ont aussi à l'esprit $\mathrm{qu}^{\prime}$ ils pourraient bien influer de manière positive sur des paramètres atmosphériques et climatiques locaux et, pourquoi pas, contribuer, même modestement, à faire baisser la teneur de l'atmosphère terrestre en GES.

\section{Des leviers d'actions complémentaires}

Notons encore que ces initiatives percent d'autant mieux qu'elles parviennent à transformer durablement les relations entre les acteurs et leur rapport avec le monde qui les entoure. Elles sont en effet tenues de s'adapter au contexte socioéconomique, territorial et culturel dans lequel elles opèrent. Leur essor dépend de leur capacité de trouver des formes de sensibilisation adaptées aux spécificités des publics auxquels elles s'adressent, et de prendre en compte aussi le fait que d'un contexte territorial et culturel à l'autre, les enjeux varient et les composantes de l'atmosphère donnent lieu à des appréciations variables. Des artistes l'ont d'ailleurs fort bien compris, quand ils s'emploient à travailler les relations aux météores et au changement climatique en s'appuyant sur les motifs culturels et symboliques propres aux différents collectifs humains (festival « Si la mer monte » de l'Île-Tudy; spectacle "Atmosphère, Atmosphère " créé en partenariat avec le PNR LoireAnjou-Touraine et qui circule dans toute la France).

Finalement, bien que limitées, fragiles, encore éclatées et disparates, ces initiatives apportent des leviers d'actions et des relais que les stratégies dédiées peuvent difficilement faire émerger. Il ne s'agit donc pas de les opposer à la planification descendante - opposition dont plusieurs auteurs (Tubiana et al., 2010 ; Berdoulay et Soubeyran, 2014) ont souligné combien elle serait défavorable au potentiel de transformation et d'innovation qui accompagne les véritables stratégies d'adaptation. Au contraire, ces initiatives peuvent assurer un véritable travail de médiation, en contribuant à la contextualisation de dispositifs, outils et mesures conçus de manière globale par rapport au changement climatique. Au demeurant, cette médiation n'a aucune raison d'être cantonnée à un échelon local ; moyennant un accompagnement adéquat, certaines initiatives pourraient très bien déployer plus largement leur fonction d'activation.

\section{Reformulation du problème et perspectives}

En croisant les observations et les résultats précédents, puis en les confrontant à la manière dont le problème climatique est habituellement formulé, des éléments significatifs pour une refondation de l'approche climatique se dégagent.

\section{La thèse du découplage science/politique brouille les signes}

Face aux menaces que font peser les changements climatiques, le déficit d'action et d'engagement politique est interprété, le plus souvent, comme « une insuffisante prise de conscience des enjeux », une " absence de réelle volonté politique ». L'échec de Copenhague, l'incapacité des États à s'engager sur des mesures fortes proviendraient, en fin de compte, du "déni de science » dans lequel les humains, pour la plupart, continueraient de se complaire. Ces différentes explications, pour séduisantes qu'elles soient en raison de leur caractère générique et de leur portée universelle, ne résistent pas à l'épreuve du local et du terrain. Acteurs privés et publics, dans les territoires, les entreprises et les autres formes d'organisations, loin d'être indifférents aux données scientifiques relatives à la réalité du changement climatique, tentent très souvent de les traduire concrètement. En prenant pour exemple la France et en suivant la chaîne descendante des plans climat-énergie, il est aisé $d^{\prime}$ attester que les efforts d'appropriation et de mise en œuvre sont réels. En outre, en France comme ailleurs, fourmillent les initiatives et les projets innovants ayant pour effet, intentionnel ou non, de contribuer à changer la donne climatique. Cela invalide dans une large mesure la thèse du découplage entre science et politique quand celle-ci laisse entendre que le problème vient de ce que les responsables politiques et la société au sens large refusent le message des sciences du climat et rechignent à prendre les mesures qui s'imposent comme si des solutions clés en main existaient déjà. Au passage, cela montre que la lutte contre le changement climatique gagnerait à accorder à certains faits ou signes sociétaux, de grande portée stratégique, autant d'attention qu'aux faits scientifiques propres au système climatique.

\section{La double nature du problème climatique}

D'où vient alors le problème ? Là encore, les retours d'expériences aux échelles locales s'avèrent fort instructifs. Ils donnent à voir les limites rapidement atteintes des stratégies dédiées d'atténuation et d'adaptation, les acteurs locaux ne parvenant pas, au-delà d'un certain stade d'engagement, à faire beaucoup plus. Pour eux, la difficulté ne se résume pas à un manque de moyens financiers et humains, parfois criant il est vrai ; ni à une carence souvent manifeste d'alternatives technologiques ; ni à l'impérieuse nécessité de préserver croissance, emplois et compétitivité - même si ces différentes contraintes et exigences existent assurément. La 
difficulté s'avère plus fondamentale, comme le suggèrent les multiples initiatives, privées et publiques, qui tentent d'aborder le problème climatique autrement, en jouant moins sur les risques et les menaces que sur les gains et les opportunités; en cherchant moins à traiter ce problème directement qu'en prenant des voies détournées ou latérales; en visant moins à supprimer une cause clairement identifiable et isolable d'un contexte qu'à transformer en profondeur un système $\mathrm{d}$ 'interactions, quitte à ce que cela prenne du temps.

D'où vient le problème alors ? De ce que le cadrage dominant du problème climatique ne rend que partiellement compte de la difficulté réelle. De prime abord et d'un point de vue global en effet, la situation peut s'énoncer selon un schéma linéaire cause-conséquences extrêmement simple : une cause unique - les émissions excessives de GES d'origine anthropique - pour une atmosphère une et indivisible, ne connaissant pas de frontière, engendre une perturbation plus ou moins forte du système climatique, avec divers effets néfastes pour les humains. La force et la simplicité de cet énoncé justifient l'approche top-down de la négociation internationale et la focalisation du débat sur des outils-solutions techniques et dédiés. Cependant, à l'échelle locale, il en va tout autrement. Le changement climatique n'est pas seulement une réalité difficilement appropriable tant elle renvoie à un vaste problème, presque insaisissable et abstrait dans la mesure où les liens de cause à effet, sur le terrain, sont difficiles à établir ; il est surtout difficilement séparable d'un ensemble de considérations, de processus et de facteurs multiples et variés, de surcroît souvent spécifiques d'un contexte local, organisationnel ou territorial ${ }^{11}$. En fin de compte, le problème du changement climatique est à l'image du climat. En s'intéressant au système-Terre dans son ensemble, les sciences de la nature sont parvenues à définir « un climat global » ou encore " un système climatique unifié », telle une entité objective et autonome : une extériorité surplombante. En pratique, cependant, pour la très grande majorité des Terriens, la notion de climat ${ }^{12}$ se décline au pluriel, ne prend véritablement sens qu'en rapport avec des circonstances géographiques précises et implique donc une multiplicité d'espaces-temps possibles (Tabeaud, 2010) ; en outre et surtout, elle renvoie à une réalité des plus immatérielles - à vrai dire, un climat ne se rencontre pas ! - tout en étant absolument consubstantielle aux sociétés humaines - influençant, aujourd'hui comme hier, les aspects les plus concrets de l'existence (milieux de vie, disponibilité alimentaire, énergies, etc.) ainsi que

11 Résultat auquel parvient aussi un groupe d'anthropologues (Barnes et al., 2013).

12 Entendu comme «la série des états de l'atmosphère audessus d'un lieu dans leur succession habituelle», le climat relève bien du concept. les dimensions politiques, culturelles, symboliques et religieuses. Il convient donc de reconnaître la double nature du problème climatique, son double visage ${ }^{13}$. D'un côté, il offre une face lumineuse, pouvant se formuler dans des termes purement objectifs, simples et universels; de l'autre, il présente une face immensément complexe $^{14}$, variable d'un territoire à l'autre en fonction des relations que les acteurs ont développé entre eux et avec leurs milieux de vie, et s'avère absolument inséparable de la subjectivité des individus et des organisations sociales. Cette double nature du problème climatique en fait un problème d'action redoutable qui oblige à considérer à nouveaux frais la question du « comment faire ». Confondre ce problème d'action avec le phénomène $d u$ changement climatique assimilé, à tort, à un "problème objectif » ne peut qu'entretenir malentendus et incompréhension réciproque.

Ainsi, l'approfondissement du problème climatique par l'écoute du terrain enseigne que le véritable découplage se situe entre acteurs globaux et acteurs locaux. Découplage qui ne doit pas être entendu comme une séparation ou une coupure absolue, mais plutôt comme une coordination encore insuffisante ou un déficit d'articulation préjudiciable à l'ensemble. En effet, dans un pays fortement administré comme la France, les stratégies et politiques climatiques définies en haut lieu ont largement percolé jusqu'à l'échelle locale ; mais en soi, leur capacité à impulser le changement espéré demeure limitée. À l'inverse, le foisonnement d'initiatives locales révèle de formidables leviers d'action en matière climatique, mais le potentiel de ces dernières n'a pas vraiment percé dans les instances globales. En somme, acteurs locaux et acteurs globaux continuent d'évoluer dans des ordres de réalité stratégique différents au regard du problème climatique; ils n'articulent pas au mieux leurs capacités d'expertise et leurs leviers d'actions respectifs. Aussi l'enjeu consiste-t-il à fluidifier la circulation ou le passage entre le global et le local, entre le ciel et la terre, rôle qui dans la tradition incombait justement à Janus, dieu des portes à double visage.

\footnotetext{
13 Une figure de la climatologie outre-Manche, le professeur Mike Hulme, connu notamment pour son ouvrage Why we disagree about climate change (2009), n'est visiblement pas indifférent à cette double nature du problème. Dès l'année universitaire 2015-2016, il ouvre au sein du département de géographie du King's College London un Master of Arts (MA) intitulé Climate Change: History, Cuture and Society qui viendra compléter un Master of Science (MSc) intitulé Climate Change: Environment, Science and Policy (http://www.kcl.ac.uk/prospectus/ graduate/climate-change-history-culture-society).

14 Différents auteurs ont tenté de dépasser la simplicité trompeuse de l'énoncé climatique, de manière indirecte (Hulme, 2009 ; Ostrom, 2009) ou encore en arguant de la nature même du problème (rapport Hartwell, Prins et al., 2010), mais il est vrai de manière insuffisamment aboutie (Aykut et Dahan, 2011).
} 


\section{Du « sauvetage du climat » à la prise en charge active de la qualité de l'air et de l'atmosphère}

$\mathrm{Au}$ travers de cet article, nous avons tenté de rendre cette perspective plus claire, sachant que beaucoup reste à faire afin de la dégager plus nettement et de la rendre effective. La nature même du problème climatique, sa complexité intrinsèque plaident en effet en faveur de la mise au jour d'un ensemble de considérations préalables ou préparatoires à sa gestion, que le terme de propédeutique $^{15}$, dans sa triple acception, permet de réunir : 1 . au sens médical, il implique d'être attentif aux symptômes et aux signes, afin de poser un bon diagnostic ; 2 . au sens pédagogique, il requiert de se donner les moyens de favoriser l'appropriation par tous d'une matière difficile, aux dimensions nombreuses; 3 . au sens philosophique, il suppose d'identifier les fondements indispensables à l'établissement d'une science et d'un art permettant de traiter avec efficacité le problème considéré. La poursuite et l'approfondissement de cet effort doivent permettre de mieux repérer les conditions et les moyens d'une meilleure articulation et d'une meilleure synergie entre capacités d'expertise et d'action aux niveaux local et global. Avec, à la clé, un potentiel de transformation considérable de l'approche climatique, pour des effets concrets sensiblement augmentés, ainsi que le pressentent différents responsables, comme Filipp Giorgi, membre historique du GIEC, lorsqu'il déclare : «[...] Nous devrions commencer à cibler d'autres publics que les gouvernements. Lorsque l'on voit les difficultés des nations à s'accorder sur des engagements de long terme, la dynamique devrait venir du "bas". Il est donc temps pour le GIEC de s'adresser à différents types $\mathrm{d}$ 'acteurs pour alimenter leurs réflexions et leurs actions et permettre une approche "bottom-up" des enjeux liés au changement climatique. ${ }^{16}{ }^{1} C^{\prime}$ est aussi, semble-t-il, le sens de la mission confiée par Laurent Fabius à la représentante spéciale pour la conférence Paris Climat 2015 (COP 21), sa fonction consistant à «mobiliser tous les acteurs pour porter un agenda positif ».

Conçue dans les seuls termes de l'imputation, la responsabilité s'accompagnait d'une double approximation : elle mettait en avant un «climat global » élevé au rang douteux de «bien commun» (Brédif et Christin, 2009 ; Brédif et Tabeaud, 2013) et tendait à confondre ce dernier avec une question énergétique, appréciée au travers d'une teneur en GES. Changer de cadre, promouvoir une approche plus positive de la responsabilité, au

\footnotetext{
15 À condition de renouer avec la richesse de ce terme et sa pluralité de sens, loin de l'usage atrophié qu'en a retenu la pédagogie.

16 Extrait d'un entretien publié dans la lettre de l'association pour la recherche et l'innovation, $\mathrm{n}^{\circ} 10$, juin 2014, «Science et société » (online: http:/ /www.air-climat.org/dossiers/scienceet-societe/interview-jean-jouzel-et-filippo-giorgi/).
}

travers de laquelle acteurs locaux et acteurs globaux puissent interagir de manière constructive suppose d'effectuer un léger pas de côté, de quitter l'abstraction du climat pour s'intéresser à la qualité de l'air et de l'atmosphère. Dès lors qu'elles ne peuvent plus être considérées comme un donné, la qualité de l'air et la qualité de l'atmosphère entrent possiblement dans la sphère des réalités susceptibles d'être prises en charge de manière active par les humains. Elles peuvent donner lieu à des projets, à toutes les échelles, même s'il est possible que dans un premier temps, il soit plus aisé de leur donner corps à des échelles locales où les responsables et acteurs auront davantage de facilité à s'accorder sur les paramètres de la qualité de l'air et de l'atmosphère qu'ils entendent améliorer et piloter ensemble.

\section{Références}

Ademe, 2013. Les représentations sociales de l'effet de serre. Résultats de l'enquête 2013, Paris (online: www.ademe.fr).

Aykut, S., Dahan, A., 2011. Le régime climatique avant et après Copenhague: sciences, politiques et l'objectif des deux degrés, Natures Sciences Sociétés, 19, 2, 144-157.

Barnes, J., Dove, M., Lahsen, M., Mathews, A., McElwee, P., Moore, F., O'Reilly, J., Orlove, B., Puri, R., Weiss, H., Yager, K., 2013. Contribution of anthropology to the study of climate change, Nature Climate Change, 3, 541-544 (online: http://dx.doi.org/10.1038/nclimate1775).

Berdoulay, V., Soubeyran, O., 2014. Adaptation, science de la durabilité et pensée planificatrice, Natures Sciences Sociétés, $22,2,114-123$.

Bertrand, F., 2013. L'institutionnalisation locale des politiques climatiques en France, in Bertrand, F., Rocher, L. (Ed.), Les territoires face aux changements climatiques: une première génération d'initiatives locales, Bruxelles, Éditions Peter Lang, Coll. Ecopolis, 25-70.

Bertrand, F., Rocher, L., 2007. Le changement climatique, révélateur des vulnérabilités territoriales? Rapport final programme Politiques territoriales et développement durable (D2RT), UMR Citeres 6173, Université de Tours (online: http:// citeres.univ-tours.fr/p_vst/contrats/D2RTrapportfinal.pdf).

Bertrand, F., Larrue, C., 2007. Gestion territoriale du changement climatique: une analyse à partir des politiques régionales. Programme GICC, UMR Citeres 6173, Université de Tours (online: http:/ /www.gip-ecofor.org/gicc/?q=node/275).

Bertrand, F. (Ed.), Richard, E., Rocher, L., Semal, L., 2012. L'adaptation au changement climatique : les défis d'une approche intégrée pour les territoires. Rapport final du projet Adap'Terr, Programme GICC, UMR Citeres 6173, Université de Tours (online: http:/ /www.gip-ecofor.org/ gicc $/$ ?q=node $/ 316$ ).

Betsill, M., Bulkeley, H., 2004. Transnational networks and global environmental governance: The Cities for Climate Protection Program, International Studies Quaterly, 48, 2, 471-493. 
Bourg, D., Whiteside, K., 2010. Vers une démocratie écologique : le citoyen, le savant et le politique, Paris, Éditions du Seuil/La République des Idées.

Brédif, H., Christin, D., 2009. La construction du commun dans la prise en charge des problèmes environnementaux: menace ou opportunité pour la démocratie?, VertigO, 9, 1 (online: http:/ / vertigo.revues.org/8489).

Brédif, H., Tabeaud, M., 2013. Entre climat et stratégie, une relation problématique, Espaces temps.net, Travaux, 23 septembre (online: http://www.espacestemps.net/ articles/entre-climat-et-strategie-une-relation-problematique).

Corfee-Morlot, J., Kamal-Chaoui, L., Donovan, M.G., Cochran, I., Robert, A., Teasdale, P.-J., 2009. Cities, Climate Change and Multilevel Governance, OECD Environment Working Papers, $\mathrm{n}^{\circ}$ 14, OECD Publishing (online: http://dx.doi.org/ 10.1787/220062444715).

CGDD/SOeS, 2012. L'empreinte carbone de la consommation des Français : évolution de 1990 à 2007, Le Point Sur, 114, MEDTL, Paris.

Committee of the Regions, European Union, 2013. Climate change adaptation: Empowerment of local and regional authorities, with a focus on their involvement in monitoring and policy design (online: http://cor.europa.eu/en/documentation/ studies/Documents/climate-change-adaptation.pdf).

Conway, E.M., Oreskes, N., 2014. L'Effondrement de la civilisation occidentale, Paris, Les Liens qui Libèrent.

Cour des comptes, 2014. La mise en œuvre par la France du Paquet énergie-climat. Synthèse du rapport de la Cour des comptes, Paris.

Dupuy, J.-P., 2004. Pour un catastrophisme éclairé. Quand l'impossible est certain, Paris, Éditions du Seuil.

Emelianoff, C., 2004. Les villes européennes face au développement durable: une floraison d'initiatives sur fond de désengagement politique, Cahier $d u$ Proses, 8, Fondation nationale des sciences politiques (FNSP), Paris.

Emelianoff, C., 2014. Local energy transition and multilevel climate governance: Lessons from two pioneer cities (Hanover, Germany, and Växjö, Sweden), Urban Studies, 51, 7, 1376-1391.

Giddens, A., 1994. Les Conséquences de la modernité, Paris, L'Harmattan.

Godard, O., 2012. Les controverses climatiques en France : la logique du trouble, in Zaccaï, E., Gemenne, F., Decroly, J.-M. (Ed.), Controverses climatiques, sciences et politique, Paris, Presses de Sciences-Po, 117-140.

Godinot, S., 2011. Les plans climat énergie territoriaux : voies d'appropriation du facteur 4 par les collectivités et les acteurs locaux?, Développement durable et territoires [En ligne], 2, 1 (online: http://developpementdurable.revues.org/8874).

Hamilton, C., 2012. Nous sommes tous des climato-sceptiques, in Zaccaï, E., Gemenne, F., Decroly, J.-M. (Ed.), Controverses climatiques, sciences et politique, Paris, Presses de SciencesPo, 221-243.
Hamilton, C., 2013. Requiem pour l'espèce humaine, Paris, Presses de Sciences-Po.

Hodson, M., Marvin, S., 2010. World cities and climate change: Producing urban ecological security, Maidenhead (UK), Open University Press.

Hopkins, R., 2010 [2008]. Manuel de transition : de la dépendance du pétrole à la résilience locale, Paris, Écosociété.

Hulme, M., 2009. Why we disagree about climate change: Understanding Controversy, Inaction and Opportunity, Cambridge (UK), Cambridge University Press.

Latour, B., 2013. Telling Friends from Foes at the Time of the Anthropocene. Texte lu à l'occasion du Colloque EHESS/ Centre Koyré/Sciences-Po Thinking the Anthropocene, 14-15 nov. 2013, in Hamilton, C., Bonneuil, C., Gemenne, F. (Eds), Thinking the Anthropocene: Rethinking Modernity in a New Epoch, London, Routledge (online: http://www.brunolatour.fr/sites/default/files/131-FRIENDS-FOESANTHROPOCENE.pdf).

Lussault, M., 2003. Article « Local », in Lévy, J., Lussault, M., (sous la Dir. de), Dictionnaire de la géographie et de l'espace des sociétés, Paris, Belin, 572-574.

MIES, 2006. Actualisation du Plan Climat 2004. Rapport du Groupe de travail collectivités locales, Ministère de l'Écologie et du Développement durable, Paris.

Oreskes, N., Conway, E.M., 2010. Les Marchands de doute, Paris, Le Pommier.

Ostrom, E., 2009. A Polycentric Approach for Coping with Climate Change, World Bank Policy Research Working Paper, 5095.

Petit, S., 2011. Le temps de demain : un collectif engagé autour du changement climatique et de ses impacts, Terrains et Travaux, 18, 103-120.

Prins, G., Galiana, I., Green, C., Hulme, M., Korhala, A., Korhala, E., Pielke Jr, R., Rayner, S., Sarewitz, D., Stehr, N., von Storch, H., 2010. The Hartwell Paper: A New Direction for Climate Policy after the Crash of 2009. Joint research paper of the Institute for Science, Innovation and Society and the MacKinder Programme for the Study of Long-Wave Events, Institute for Science, Innovation and Society, Oxford.

Rosanvallon, P., 2009. Sortir de la myopie des démocraties, Le Monde, 7 décembre.

Serres, M., 2009. Temps des crises, Paris, le Pommier.

Stern, N., Calderon, P. (Ed.), 2014. Better Growth, Better Climate: The New Climate Economy Report (online: http:// newclimateeconomy.report/).

Tabeaud, M., 2010. Les espaces-temps des climats, Historiens et Géographes, 411, 117-124.

Tabeaud, M., 2011. in Veyret, Y., Legoix, R. (Eds), Atlas des villes durables. Écologie, urbanisme, société : l'Europe est-elle un modèle?, Paris, Autrement.

Tubiana, L., Gemenne, F., Magnan, A., 2010. Anticiper pour s'adapter: le nouvel enjeu du changement climatique, Paris, Pearson Éducation. 